\title{
Quantification of Urushi Dissolution by Ultraviolet Irradiation for an Urushi - based Electronic Circuit
}

\author{
Yu MYOJIN, ${ }^{\mathrm{a}, *}$, Yuki HASHIMOTO ${ }^{\mathrm{b}}$ \\ aDepartment of Intelligent Interaction Technologies, University of Tsukuba, Tsukuba-shi, Ibaraki, Japan \\ ${ }^{b}$ Engineering Mechanics and System, University of Tsukuba, Tsukuba-shi, Ibaraki, Japan \\ *myojin@vrlab.esys.tsukuba.ac.jp
}

\begin{abstract}
Urushi (Japanese lacquer) has many interesting features, such as electrical insulating capabilities and vulnerability with respect to ultraviolet light (UV) exposure. Here, we describe an urushi-based multilayered electronic circuit (urushi circuit) that is created using an UV laser to locally dissolve the urushi layers. This allows the layers to be connected via the opening/groove created by the UV exposure. Experiments were performed to determine the effect of UV irradiation on the amount of urushi dissolved via variation in the irradiation spot diameter. The amount of urushi dissolved was directly proportional to the UV intensity and irradiation duration. Based on our data, we created a model to describe urushi dissolved to facilitate good reproducibility and controllability for multilayered urushi circuit design.
\end{abstract}

Keywords: Electronic circuit, Ultraviolet light, Urushi.

\section{Introduction}

The many advances in electronic device technology and wearables have greatly affected the quality of everyday life, with these devices increasingly becoming more of a necessity. Wearable devices are more prevalent in health management $(1,2)$. Some robots communicate with users to provide comfort (3). For long-term use, these devices should be resilient against severe operating environments, which may include water, chemical, and/or shock exposure. Moreover, the technology must be safe for humans, particularly those who are sensitive to fluctuations in normal device operation. Any insufficiencies may adversely affect device functioning, and ultimately, the health of the user.
One particular case in point is the LG G Watch. A malfunction in the design led to charging pin corrosion and the inability to charge the device (4). To resolve these problems, LG was required to revise the main body software, such that the G Watch could not power on unless it was plugged into a charger. Fitbit Force is another example, in which allergic skin reactions (rashes, peeling skin, and blisters) were reported by users due to nickel in the stainless steel, materials used for the strap and adhesives during assembly, and bacteria accumulation (5). Fitbit recalled their product and gave a full refund to users.

The underlying cause of the issues described is material-related. Conventionally, electronic devices use many materials to protect electronic circuitry, which is seemingly the most vulnerable part of the component. As such, much attention has been given to improving the strength and tolerance of devices against various environmental hazards (e.g., water, corrosives, and shock). However, the introduction of wearable device technology has highlighted the shortfalls of the component materials with regard to the health of the user. These problems affect users of all ages, with some being more at risk due to health conditions. In addition, the problems are compounded by the variability in the design and fabrication of the devices.

Urushi (Japanese lacquer) is a natural material used in traditional Japanese crafts that offers not only decorative beauty but also many useful features, including resistance to heat, acid, water, and humidity. It is an adhesive, but has also been used for protection against insects and electrical insulation (6). Moreover, because urushi can be applied to various bases, such as metal, glass, plastic and wood, to support and protect three-dimensional structures, it provides more flexibility in terms of material choice and structural integrity. Thus, urushi has been extensively used in daily 
necessities, such as tableware and decorative ornaments, as well as in ship lacquer and building supplies (Table 1.). As an added value, urushi offers a familiar depth and beauty, and has a unique feel (7).

Table 1. Urushi features.

\begin{tabular}{|l|c|}
\hline Water resistance & Good \\
\hline Chemical resistance & Good \\
\hline Electrical resistance & Good \\
\hline Rust resistance & Good \\
\hline Antiseptic properties & Good \\
\hline Hardness & Same hardness as iron \\
\hline Insect-repellent properties & Good \\
\hline Antimicrobial properties & Good \\
\hline Heat resistance & $120 \sim 300^{\circ} \mathrm{C}$ \\
\hline Breathability & Good \\
\hline Environmental safety & Pollution-free \\
\hline Human safety & Hypoallergenic \\
\hline
\end{tabular}

Here, we propose a safe, durable, eco-friendly electronic circuit using urushi. Ultraviolet (UV) irradiation is used to locally dissolve urushi layers, effectively connecting the layers via the dissolved material. In the present paper, we explain the proposed method, and confirm the relationship between the UV light intensity and the amount urushi dissolved.

\section{Methods and Purpose}

Urushi is already used to coat many goods in regular use, as well as furniture. It is possible to add circuit functionality to a lacquered item without changing its appearance, at a low cost. Due to its enhanced strength and resilience, urushi circuit can also be used to downsize electronic devices, without the need for additional protection.

Here, we describe the first steps in urushi circuit construction. To improve device performance in a limited amount of space, we used laminated urushi layers. In a multilayered circuit, design, openings or grooves for wiring are required to electrically connect individual layers. Conventional board-processing techniques commonly use physical methods, such as drilling or thermolysis via lasers $(8,9)$. However, the thin, flexible board material is susceptible to damage from mechanical stress and heat. Chemical etching using has also been used to remove material. However, urushi is chemically resistant.

Recent studies have shown that urushi is sensitive to UV light exposure (6). In the present study, urushi's sensitivity to UV light was used to fabricate a multilayered circuit (hereafter, urushi circuit), as shown in Fig. 1. In addition, there are some prototypes using the urushi circuit (Fig. 2) (10).

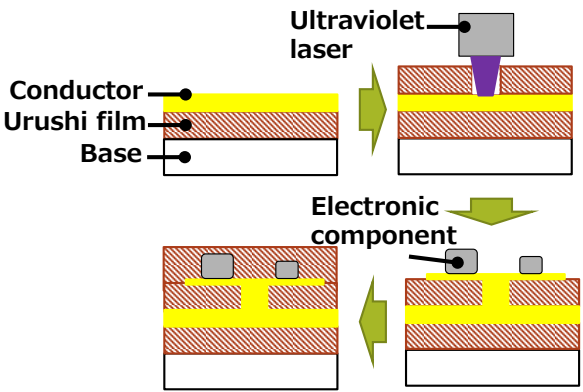

Fig. 1. Fabrication of an urushi circuit.

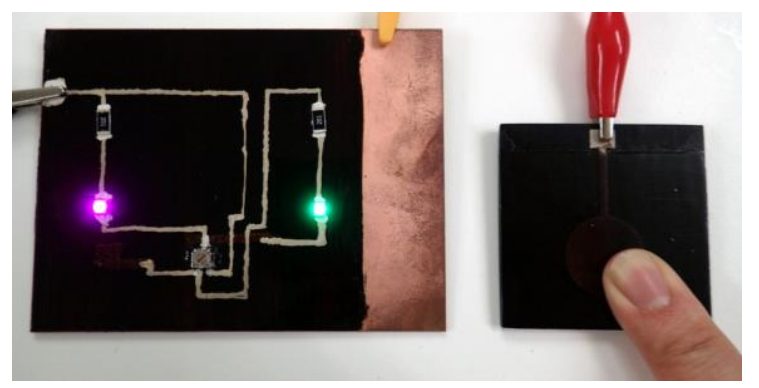

Fig. 2. A two-layer urushi circuit and a touch sensor with the urushi circuit (10).

This processing method can potentially improve fabrication precision by controlling the UV light intensity via the spot diameter and irradiation duration. In addition, this non-contact process is energy efficient due to its use of a chemical reaction, as opposed to mechanical material removal. Thus this method can support many kinds of base materials.

We hypothesized that the dissolution rate of urushi should be related to the UV irradiation intensity. We attempted to quantify this relationship, in an effort to establish reproducibility in the material dissolution process. A dissolution model was created, based on our results.

\section{Experimental Preparation}

\subsection{Test Piece Fabrication}

We lacquered urushi onto a copper plate (dimensions: $100 \times 100 \times 4 \mathrm{~mm})$ to create the test piece. The fabrication procedure for the test piece (Fig. 3) is given below.

1. Dust and oil were removed from the copper plate.

2. The thickness of the copper plate was measured using a laser displacement meter.

3. Urushi was lacquered onto the copper plate.

4. The copper plate was placed in a 
temperature/humidity controlled box, maintained at $25^{\circ} \mathrm{C}$ and $60-80 \%$ humidity.

5. The urushi coating was allowed to harden.

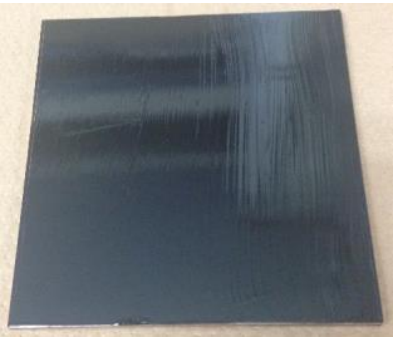

Fig. 3. Urushi test piece.

\subsection{Experiment System}

Figure 3 shows the experimental set-up, which consisted of a surface plate made of stone, an UV laser (405 $\mathrm{nm}, 200 \mathrm{~mW}$ ), a laser displacement meter (LK-G30, KEYENCE), and support plates. The test piece was placed onto a surface plate with $\mathrm{x}-\mathrm{y}$ adjustability. The UV laser and laser displacement meter were also placed on support plates to ensure accurate positioning and repeatability in the intensity and material dissolution measurements (Fig. 4-1). The distance between the test piece and the UV laser was 5 $\mathrm{cm}$ (Fig. 4-2). Note that the irradiated area was surrounded by masking tape (Fig. 5).
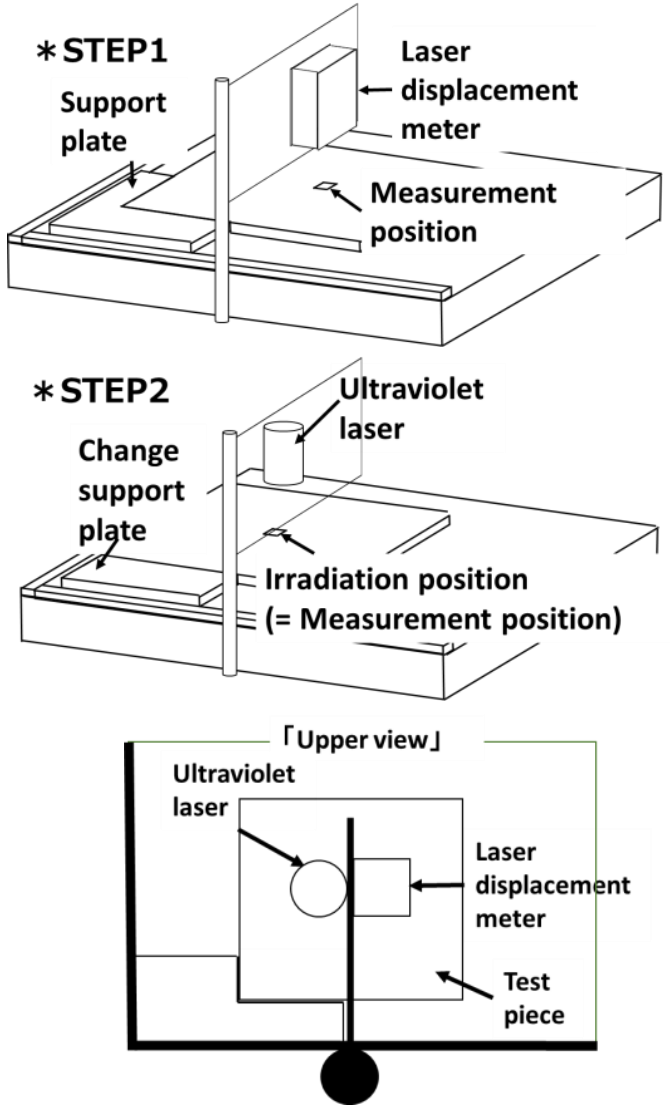

Fig. 4-1. Experimental set-up for the test piece and measurement equipment.

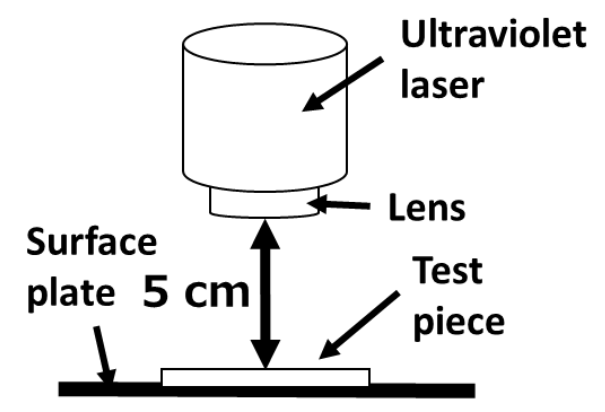

Fig. 4-2. Experimental set-up for UV laser.

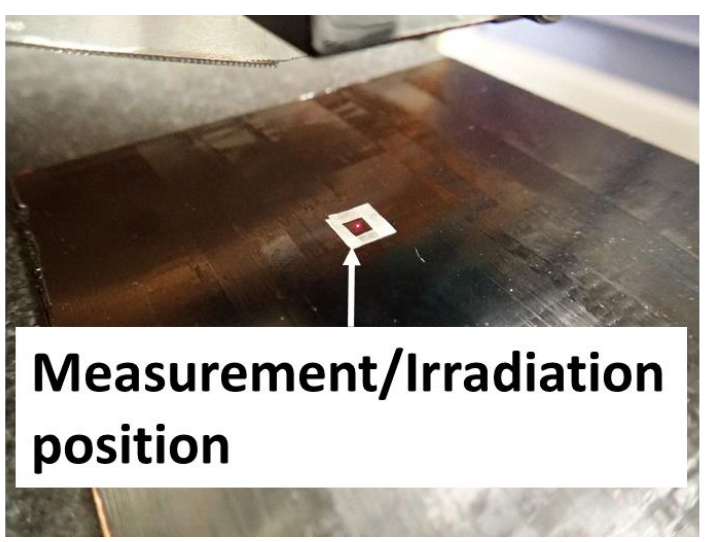

Fig. 5. Measurement/irradiation position.

\section{Experimental Evaluation}

\subsection{Hypothesis}

A recent study (6) showed that urushi's dissolution was proportional to the UV intensity. Given a constant output from the UV laser, the strength of the UV light per unit area is inversely proportional to the irradiation spot diameter of the UV laser (i.e., UV intensity : irradiation spot diameter $=$ dissolution speed : irradiation duration). The irradiation duration per trial per irradiation spot diameter (Table 2) was based on this relationship. In this experiment, three irradiation spot diameters were compared.

Table 2. Irradiation duration based on the spot diameter.

\begin{tabular}{|l|l|l|l|}
\hline Irradiation spot diameter $(\mathrm{mm})$ & 1.0 & 1.5 & 2.0 \\
\hline Area (from ratio) & 1 & 2.25 & 4 \\
\hline Irradiation duration per trial (s) & 60 & 135 & 240 \\
\hline
\end{tabular}

\subsection{Purpose of the Experiment}

We attempted to confirm our hypothesis based on the repeatability of urushi material dissolution for the same spot diameter, followed by a comparison of the material dissolution given different irradiation spot diameters. The targeted error range $10 \%$. Thus, the experiments were 
divided into two parts as specified below.

\section{Analysis based on identical irradiation spot diameters}

Given the same irradiation spot diameter, we attempted to verify the repeatability of urushi dissolution.

2. Comparative analysis of the irradiation spot diameters

A comparison of the experimental results and theoretical results (Table 2) was used to confirm the consistency of urushi dissolution for model generation.

\subsection{Experiment Description}

The test piece was exposed to UV irradiation, using spot diameters of $1.0,1.5$, or $2.0 \mathrm{~mm}$; the irradiation times are specified in Table 2 for the given spot diameters. The depth of dissolution was measured using a laser displacement meter for each irradiated test piece. The experimental procedure is outlined below.

1. The distance between the surface plate and the laser displacement meter was measured and set as a reference $(x(0))$.

2. The test piece was placed on the surface plate and irradiated with UV light.

3. The thickness of the test piece was measured with respect to the reference value $(x(0))$.

4. The procedure was repeated 10 times. The results, excluding the maximum and minimum values, were averaged and the standard deviation (SD) for the material dissolution rate was calculated.

Twelve trials were performed using the procedure described above, for each irradiation spot diameter. These twelve trials made up one set, for a total of three sets.

\subsection{Experimental Results}

Graphs of the experimental results (Fig. 6-7) are placed at the end of this paper.

Analysis based on identical irradiation spot diameters

Fig. 6-1, 6-2, and 6-3 show the following, respectively:

- Cumulative amount of urushi dissolved.

- Amount of urushi dissolved per trial, with linear approximation.

- Difference between the maximum and minimum urushi dissolution quantities per trial.

The SD ranged from $0.15-0.35 \mu \mathrm{m}$. The measurement error was set to $\pm 0.2 \mu \mathrm{m}(\sim \pm 1 \mathrm{SD})$. The experimental error was set as $\pm 0.4 \mu \mathrm{m}(\sim \pm 2 \mathrm{SD})$; this error was included in the difference in the cure degree and urushi components. The tolerance range was based on the measurements and experimental error. If the experimental measurement was $<0.4 \mu \mathrm{m}$, then the data were deemed to have some repeatability. Thus, referring to the graphs of (3) in Fig. 6-1, 6-2, and 6-3, we decided that if measured values less than $0.4 \mu \mathrm{m}$ appeared more than twice and subsequent values were less than $0.8 \mu \mathrm{m}$, then the results were repeatable. A linear approximation was made based on these results.

From graph (1) of Fig. 6-1, 6-2, and 6-3, only a slight difference in the cumulative dissolution quantity was observed among the three UV irradiation spot diameters tested. Graph (3) shows repeatability in the dissolution results among the spot diameters tested (Fig. 6.1-6.3) for the fourth, second, and seventh identified areas. Graph (2) (Fig. 6.1-6.3) shows a linear approximation of dissolution quantities, with similar inclinations among test pieces exposed to various spot diameters (Table 3: |error per trial| $<0.4 \mu \mathrm{m}$ (measurement error)). The processing precision was $\sim 10 \%$ for a $10 \mu \mathrm{m}$ resolution, based on the error analysis results for urushi's dissolution (Table 3).

Table 3. Change in urushi dissolution amounts, average dissolution within the tolerance range, and associated error at $10 \mu \mathrm{m}$.

\begin{tabular}{|l|r|r|r|}
\hline & $\begin{array}{c}\text { Error per trial } \\
{[\mu \mathrm{m}]}\end{array}$ & $\begin{array}{c}\text { Average dissolution quantity in } \\
\text { the tolerance range }[\mu \mathrm{m}]\end{array}$ & $\begin{array}{l}\text { Error at the time of the } \\
10 \mu \mathrm{m} \text { resolution }[\mu \mathrm{m}]\end{array}$ \\
\hline$\Phi 2.0 \mathrm{~mm}$ & -0.0583 & 0.624 & 0.935 \\
\hline$\Phi 1.5 \mathrm{~mm}$ & -0.0856 & 0.808 & 1.059 \\
\hline$\Phi 1.0 \mathrm{~mm}$ & -0.0307 & 0.666 & 0.461 \\
\hline
\end{tabular}

Thus, our results indicate that similar amount of urushi were dissolved by UV irradiation of the same intensity, with high reproducibility for a given irradiation spot diameter.

\section{Comparative analysis of irradiation spot diameters}

If the difference between the maximum and minimum values of each trial set is within tolerance, then the dissolution amount should be the same regardless of the irradiation spot diameter. For this analysis, different test pieces were used for each trial set.

Fig. 7-1, 7-2, and 7-3 show the following, respectively:

- Cumulative amount of urushi dissolved.

- Amount of urushi dissolved quantity per trial, with linear approximation.

- Difference between maximum and minimum urushi dissolution quantities per trial. 
In Fig. 7-1, 7-2, and 7-3, graph (1) shows a small cumulative error for the first and second trial sets; however the third trial set shows twice the error of that in the first and second trial sets. Graph (2) shows big spikes in the third trial set this was attributed to possible dust contamination. In graph (3), we established the measurement error, using an approach similar to that for Figure 5 for the given tolerance. All of the trial sets settled into the tolerance range in the third (Fig. 7-1.), eighth (Fig. 7-2), and sixth (Fig. 7-3) trials. Thus, these areas support our hypothesis that if the difference between the maximum and minimum values of each trial set is within the tolerance, then the dissolution amount should be the same, regardless of the irradiation spot diameter.

However, the initial trials did not support this hypothesis; this was attributed to possible dust contamination, inconsistencies in UV laser operation, and/or deflection by urushi constituents.

\section{Discussion}

From our results, in the latter trials repeatable amount of urushi were dissolved that were independent of the UV irradiation spot diameter. Based on these trials, we can linearly approximate the dissolution amount per trial to create a control model for the dissolution quantity as a function of UV intensity and irradiation duration.

However, several of the initial trials showed a greater difference. One possible cause may have been inclusion of dust in the urushi. Before urushi lacquering, we filtered out the dust and cleaned the copper surface. However, the copper surface or urushi may have collected dust from the air. Thus, in future experiments, a clean environment, such as a glove box and draft chamber should be used.

Inconsistencies associated with UV laser operation may also have originated from impurity formation with dissolution. This may be resolved by using a spot diameter $<1.0 \mathrm{~mm}$ and controlling the output via the duty ratio. It may also be possible to remove impurities using an air blower during UV irradiation of the test piece. These adjustments will be made in future experiments.

\section{Conclusion}

In the present paper, we proposed a safe, eco-friendly, durable electronic circuit made from urushi, a natural material known for its resilience and resistance to caustic substances. The use of urushi provides a solution to several wearable electronic device shortcomings, in terms of structural integrity, electrical insulation, corrosion resistance, and hypoallergenic safety. In the present study, a multilayered urushi electrical circuit was fabricated using UV irradiation to dissolve the urushi material locally to create a groove or opening for an electrical connection. We verified the relationship between UV irradiation intensity and the amount of urushi dissolution. We examined the hypothesis that the amount of urushi dissolved is proportional to the UV intensity, based on the irradiation spot diameter. Our results showed that in the latter trials the amounts of urushi dissolved were repeatable, independent of the irradiation spot diameter. Based on these trials, we linearly approximated the dissolution amount per trial, to create a control model for the amount of urushi dissolved as a function of the UV intensity and irradiation duration. In future experiments, we plan to improve the experimental environment for more precise processing of urushi. We will attempt to establish a conducting urushi surface for multilayer circuit design, with the purpose of creating an urushi prototype circuit.

\section{Acknowledgments}

This work was supported by The Foundation for Technology Promotion of Electronic Circuit Boards.

\section{References}

(1) NIKE, Inc., "NIKE+FUELBAND", http://www.nike.com/us/en_us/c/nikeplus-fuelband

(2) Healbe GoBe: The Only Way to Automatically Measure Calorie Intake https://healbe.com/

(3) JIBO, The World's First Social Robot for the Home. https://www.indiegogo.com/projects/jibo-the-world-s-fi rst-social-robot-for-the-home\#/story

(4) LG Looks The Other Way Over G Watch Charging Pin Corrosion

http://www.androidheadlines.com/2014/07/lg-looks-wa y-charging-pin-corrososion.html

(5) Fitbit Does More Than Track Fitness, It Also Leaves a Rash for Some

http://abcnews.go.com/blogs/headlines/2014/01/fitbit-d oes-more-than-track-fitness-it-also-leaves-a-rash-for-so mel

(6) Yuki Hashimoto, and Naoya Koizumi: "Basic study of development for Japan-based electronic circuit." 
Transactions of the Society of Instrument and Control Engineers, vol. 51, issue 1, pp. 64-71

(7) Kasho YOTSUYANAGI, Urushi I (Japanese). HOSEI UNIVERSITY PRESS, 2006

(8) Mitsubishi Electric, Laser Processing Machines https://us.mitsubishielectric.com/fa/en/products/process ing-machines/laser-processing-machines

(9) MITS, High Precision PCB prototyping processing, http://www.mitspcb.com/edoc/epri.htm
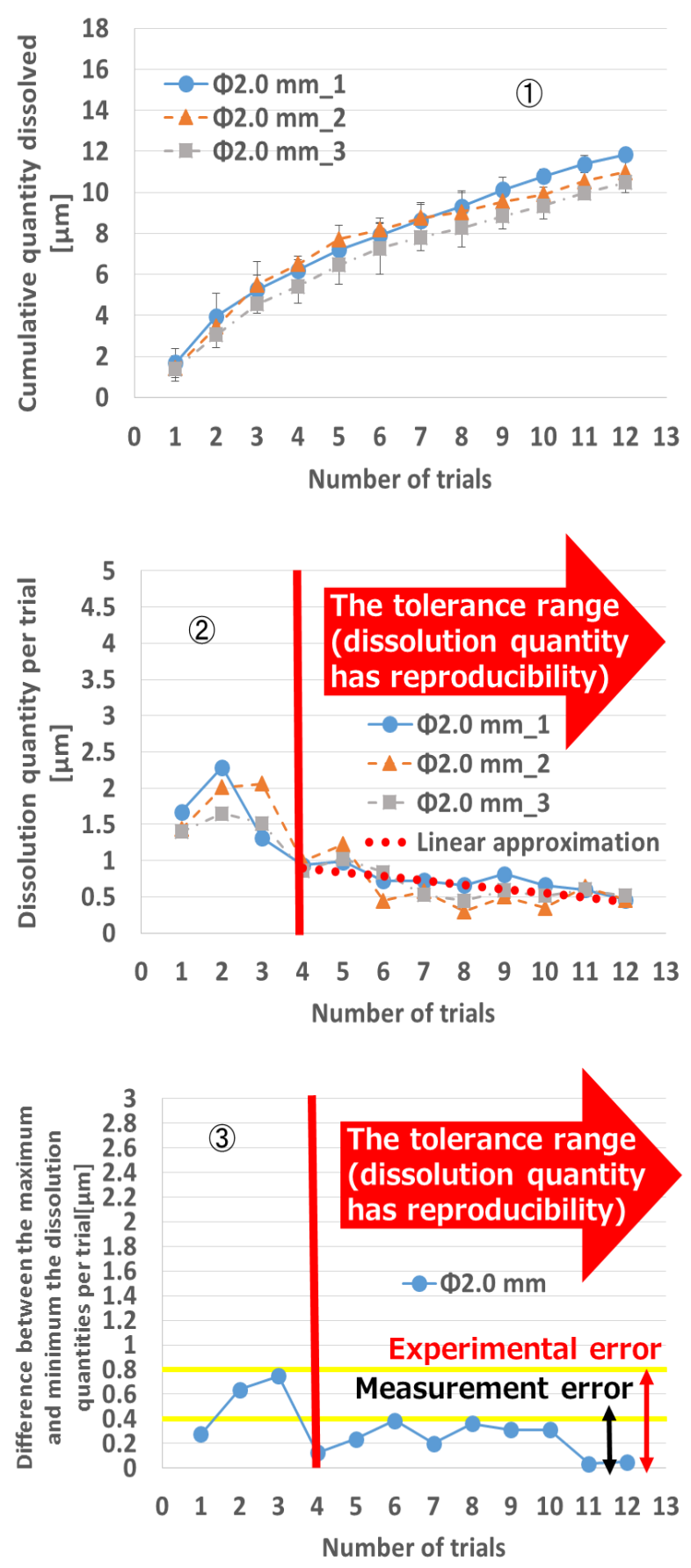

Fig. 6-1. Urushi material dissolution results for an UV irradiation spot diameter of $2.0 \mathrm{~mm}$
(10) Yuki Hashimoto, Naoya Koizumi, Yu Myojin, Buntarou Shizuki, Nobuko Hanada: "Urushi Circuit -Traditional Craft for HCI-", ACM CHI2015 Symposium on Emerging Japanese HCI Research Collection, Seoul/Korea, 2015/4/18.
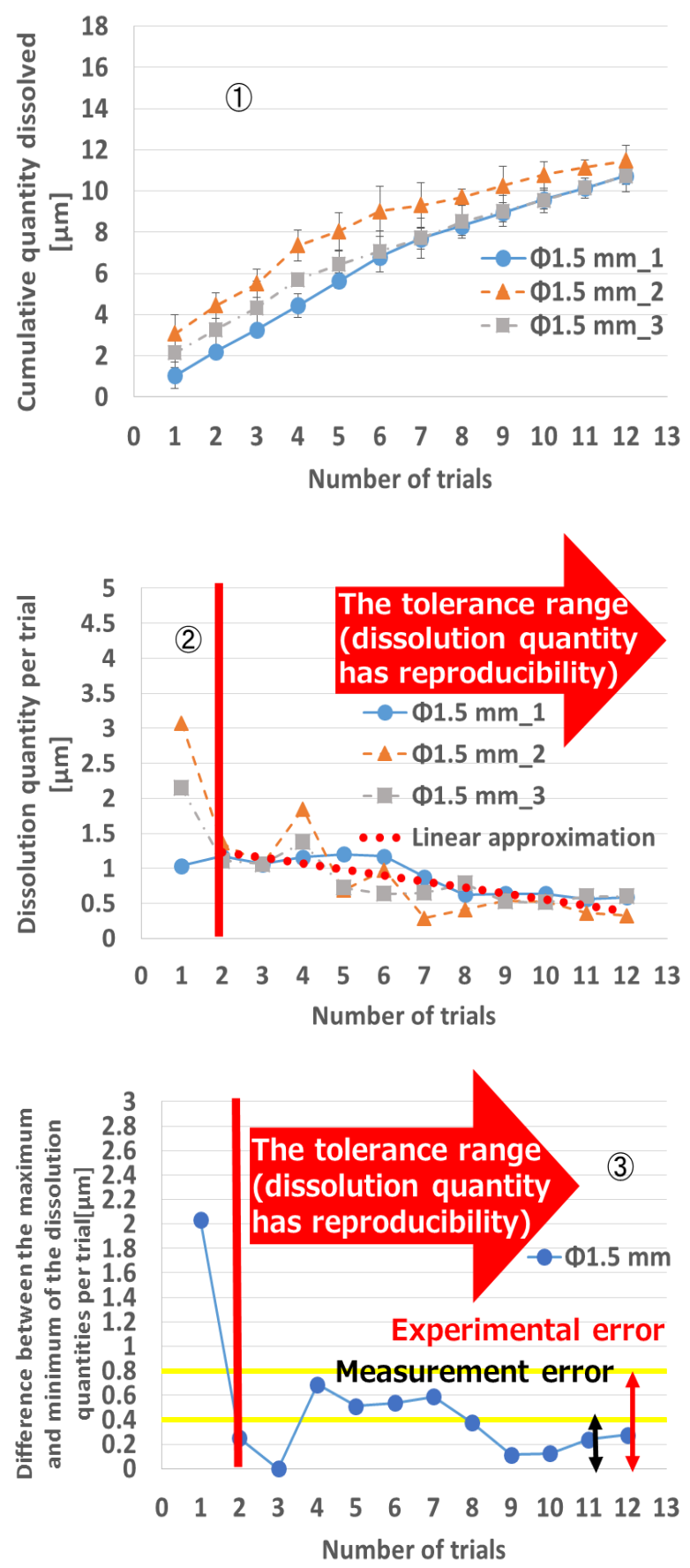

Fig. 6-2. Urushi material dissolution results for an UV irradiation spot diameter of $1.5 \mathrm{~mm}$. 

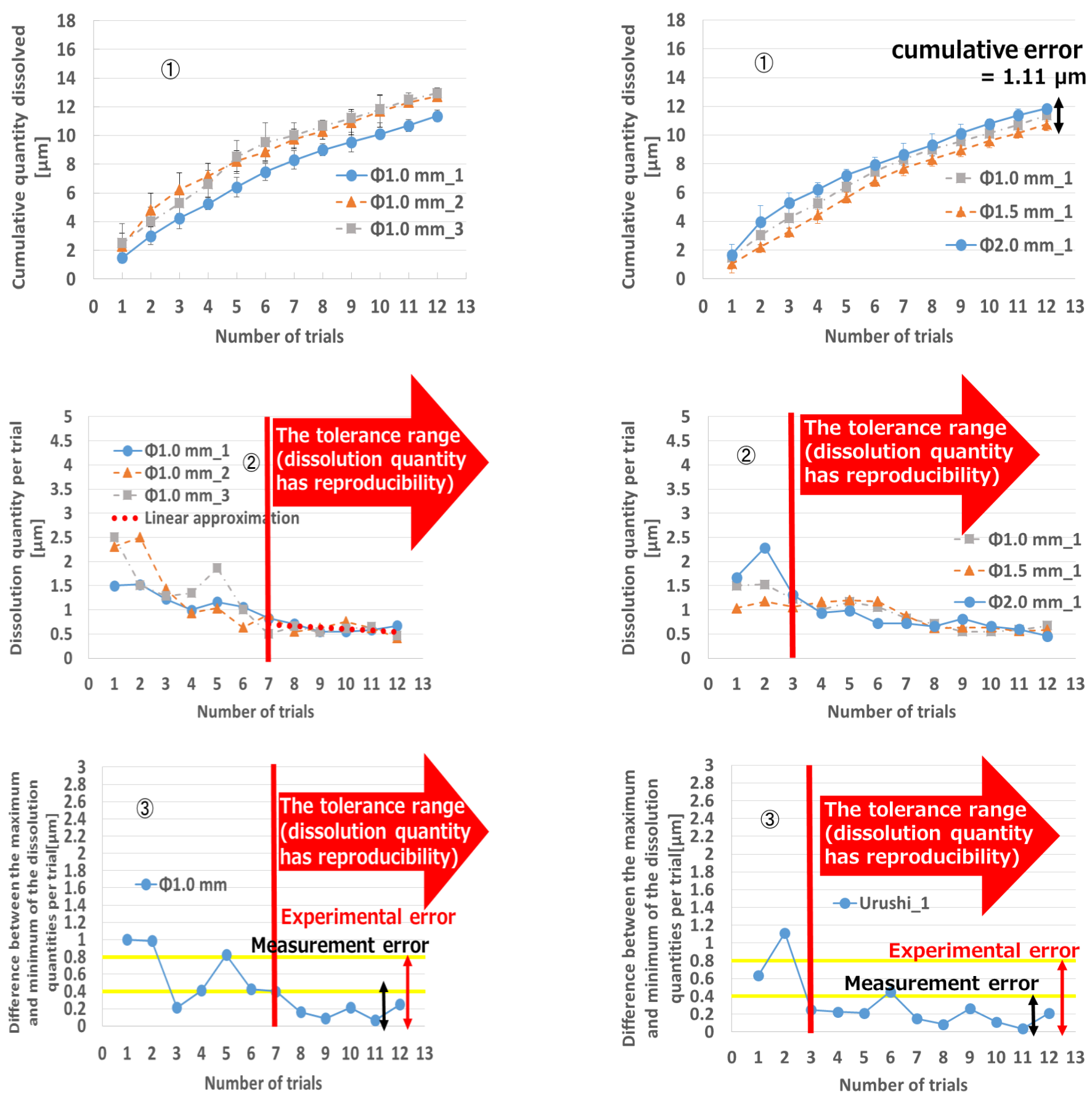

Fig. 6-3. Urushi material dissolution results for an UV irradiation spot diameter of $1.0 \mathrm{~mm}$.

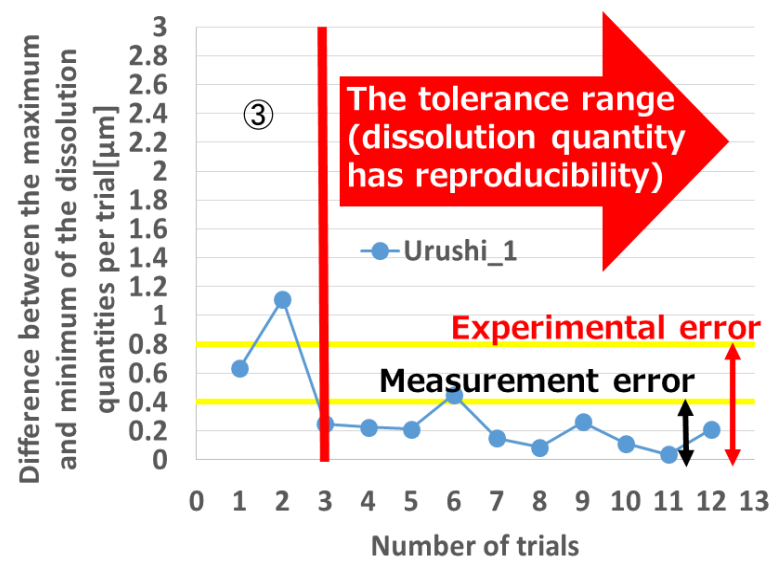

Fig. 7-1. Urushi material dissolution results for first trial set. 

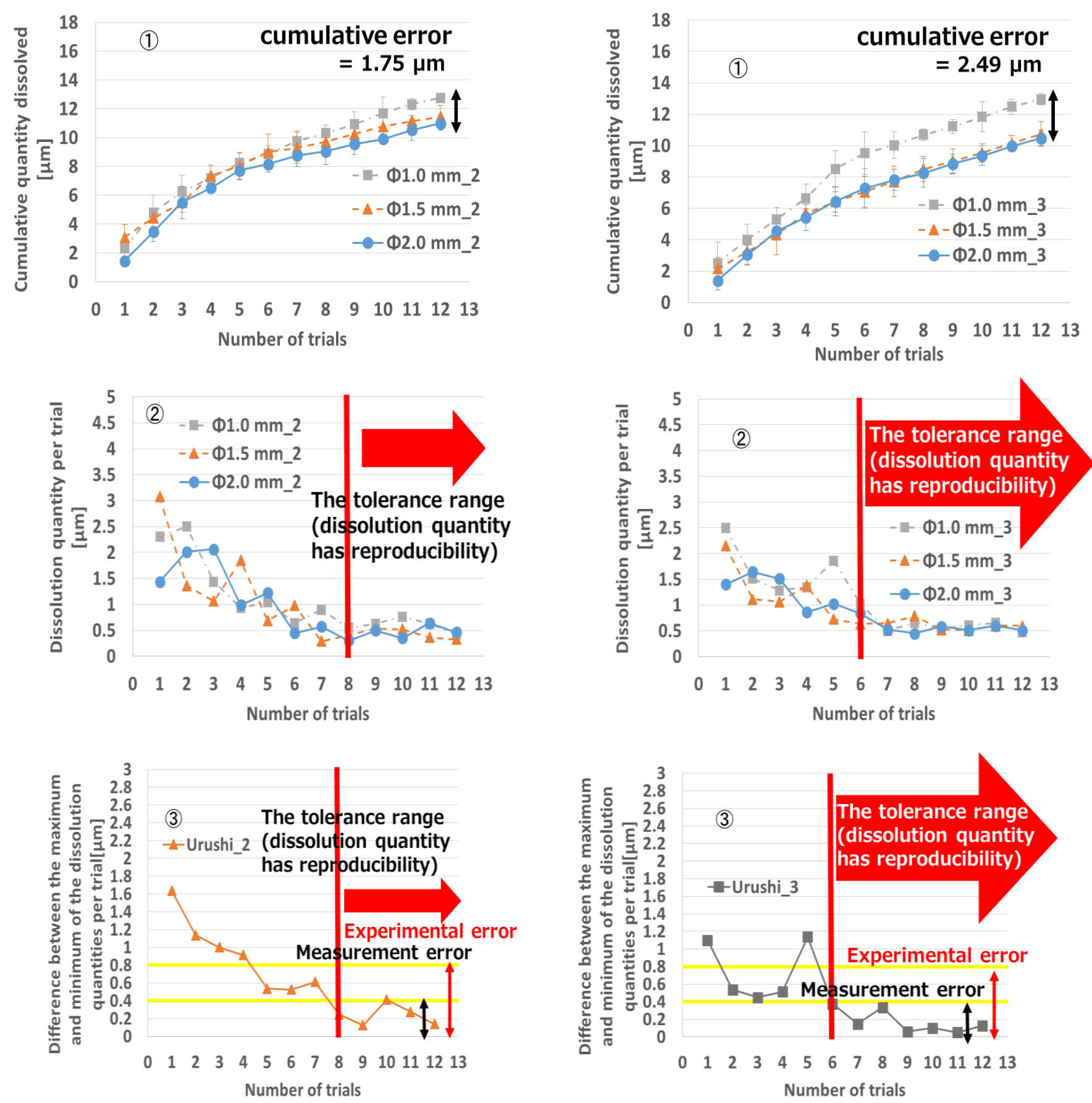

Fig. 7-2. Urushi material dissolution results for second trial set.

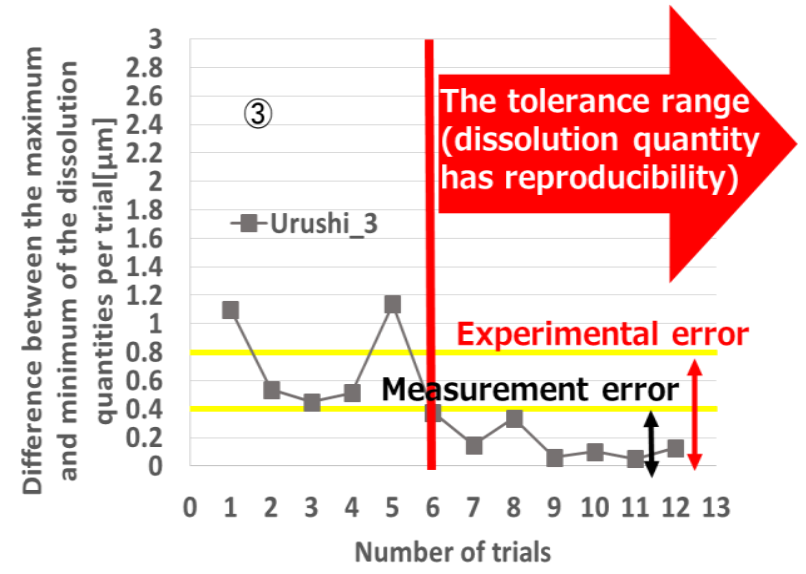

Fig. 7-3. Urushi material dissolution results for third trial set. 